\title{
TELESSAÚDE DURANTE A PANDEMIA DA COVID-19 NO BRASIL E A ENFERMAGEM
}

Juliana Dias Reis Pessalacia. Doutorado e Pós Doutorado em Enfermagem em Saúde. Docente associada Il e Coordenadora da Unidade de Telessaúde do Campus de Três Lagoas (CPTL) da Universidade Federal de Mato Grosso do Sul (UFMS). E-mail: juliana@pessalacia.com.br

Em dezembro de 2019, relataram-se vários casos de pneumonia de causa desconhecida em Wuhan, província de Hubei, China. Rapidamente essa pneumonia disseminou-se para outras províncias e países em todo o mundo. Em janeiro de 2020, identificou-se um novo coronavírus, nomeado como 2019nCoV pela Organização Mundial da Saúde (OMS) ${ }^{(1)}$, a qual declarou surto e Emergência de Saúde Pública de Interesse Internacional (ESPII) decorrente do mesmo. Em fevereiro de 2020, o novo vírus foi renomeado como Síndrome Respiratória Aguda Grave Coronavirus-2 (SARS-CoV-2) pelo Comitê Internacional de Taxonomia de Vírus (ICTV) e a OMS anunciou a doença epidêmica causada por este vírus como a Doença do Coronavírus 2019 (COVID-19) ${ }^{(2)}$.

No Brasil, atuou-se imediatamente após a detecção de rumores sobre a doença emergente. Em janeiro de 2020 acionou-se a Secretaria de Vigilância em Saúde (SVS) por meio do Ministério da Saúde (MS), buscando-se a harmonização, o planejamento e a organização das atividades com os atores envolvidos e o monitoramento da situação epidemiológica no país. Mobilizou-se diversos setores do governo e diversas ações foram implementadas, incluindo a elaboração de um plano de contingência. Em fevereiro de 2020, a infecção humana pelo novo coronavírus foi declarada Emergência em Saúde Pública de Importância Nacional (ESPIN) $)^{(3)}$.

Confirmou-se o primeiro caso de COVID-19 no Brasil em 26 de fevereiro de 2020 e, em menos de um mês após a confirmação do mesmo, já havia transmissão comunitária em algumas cidades. Em 17 de março de 2020, detectou-se o primeiro óbito no país e no dia 20 de março de 2020 foi reconhecida a transmissão comunitária em todo o território nacional ${ }^{(3)}$.

A doença progrediu de forma muito rápida e tem representado um verdadeiro desafio para todo o mundo. Considerando-se que em muitos países a capacidade de atendimento usual nos serviços de saúde foi excedida, os governos adotaram o distanciamento social como principal estratégia para reduzir e mitigar o avanço da epidemia. A partir de então, o Telessaúde tornou-se uma importante estratégia no combate à pandemia, dando suporte aos sistemas de saúde, principalmente nas áreas de saúde pública, prevenção e práticas clínicas, assim como em outros setores como o teletrabalho e o apoio à formação e Educação Permanente em Saúde (EPS) ${ }^{(4)}$. 
Assim, nota-se que a preocupação com o avanço da doença despertou o interesse nos governos e profissionais de saúde em explorar o potencial do uso de Tecnologias da Informação e Comunicação (TIC), buscando-se enfrentar os diversos desafios decorrentes da pandemia. A TIC e sua integração em sistemas de prestação de cuidados em saúde possibilitam oportunidades de melhoria do atendimento clínico, principalmente nos casos em que a distância é um fator $\operatorname{crítico}^{(5)}$.

No contexto brasileiro, o Programa Telessaúde Brasil Redes teve início em 2007, expandindo-se em 2011 para diversos estados do país ${ }^{(6)}$. O modelo de Telessaúde adotado no Brasil baseia-se na conexão das universidades com a Atenção Primária a Saúde (APS) por meio de atividades de Tele-educação e Teleassistência, visando principalmente ao fortalecimento da Estratégia de Saúde da Família (ESF) ${ }^{(7)}$. A atuação dos diversos núcleos de Telessaúde nos diferentes estados, em parceria com as universidades tem se mostrado uma importante estratégia no enfrentamento da pandemia, por possibilitar uma menor circulação de pessoas que buscam atendimento nos serviços de saúde, reduzindo-se o risco de contaminação e propagação da doença( ${ }^{(8)}$.

O Telessaúde envolve diferentes abordagens, as quais encontram-se sumarizadas no quadro abaixo:

\begin{tabular}{|c|c|}
\hline Aplicações da telessaúde & Atividades \\
\hline Teleconsultoria & $\begin{array}{l}\text { Consulta registrada e realizada entre trabalhadores, profissionais e gestores da área de saúde, objetivando } \\
\text { esclarecer dúvidas sobre procedimentos clínicos, ações de saúde e questões relativas ao processo de trabalho. }\end{array}$ \\
\hline Telediagnóstico & $\begin{array}{c}\text { Utilização das TIC em serviços de apoio ao diagnóstico por meio de distâncias geográficas e/ou temporais, que } \\
\text { inclui telerradiologia, teleECG, teleespirometria, telepatologia etc. }\end{array}$ \\
\hline Telemonitoramento & $\begin{array}{l}\text { Monitoramento à distância de parâmetros de saúde e/ou doença de pacientes, incluindo coleta de dados } \\
\text { clínicos, transmissão, processamento e manejo por profissional de saúde. }\end{array}$ \\
\hline Telerregulação & $\begin{array}{l}\text { Ações em sistemas de regulação, avaliação e o planejamento das ações, fornecendo à gestão uma inteligência } \\
\text { reguladora operacional. Possibilita a redução nas filas de espera no atendimento especializado. }\end{array}$ \\
\hline Teleeducação & Aulas, cursos ou disponibilização de objetos de aprendizagem interativos sobre temas relacionados à saúde. \\
\hline Segunda opinião formativa & $\begin{array}{l}\text { Resposta sistematizada, construída com base em revisão bibliográfica das melhores evidências científicas a } \\
\text { perguntas originadas das teleconsultorias. }\end{array}$ \\
\hline Teleconsulta & $\begin{array}{l}\text { Realização de consulta médica ou de outro profissional de saúde à distância por meio de TIC, que até a } \\
\text { epidemia só era permitida, no Brasil, pelo Conselho Federal de Medicina em situações de emergência. }\end{array}$ \\
\hline
\end{tabular}

ECG: eletrocardiograma; TIC: tecnologias de informação e telecomunicação .

Fonte: Caetano et al. ${ }^{(8)}$

Vale ressaltar que Telessaúde e Telemedicina são termos distintos, à medida que, o Telessaúde se refere a um escopo mais amplo de serviços remotos de assistência do que a Telemedicina. A Telemedicina se refere especificamente a serviços clínicos remotos, já o Telessaúde pode se referir a serviços não clínicos remotos, como treinamento de fornecedores, reuniões administrativas e educação continuada, além de serviços $\operatorname{clínicos}^{(9)}$, não restritos à classe médica, abrangendo-se outras categorias profissionais, tal como a Enfermagem.

No contexto de pandemia da COVID-19, torna-se essencial o papel da Enfermagem nas ações de Telessaúde. A Enfermagem tem sido desafiada a inovar e ampliar o escopo de sua prática durante a 
pandemia, buscando-se novas possibilidades para atender ao aumento da demanda de pacientes aos serviços e para reduzir as exposições ao vírus ${ }^{(10-11)}$. O conceito de Telessaúde em Enfermagem tem se desenvolvido recentemente e abrange a atuação dos profissionais de enfermagem que cuidam de pacientes à distância, por meio das TIC. Neste contexto, o papel da Enfermagem inclui a execução de avaliações e prestação de serviços remotamente, além do auxílio para que pacientes possam participar de uma consulta por meio de TIC com um profissional de saúde fisicamente localizado em outro lugar ${ }^{(11)}$. Outra possibilidade de atuação no cenário das unidades de saúde da APS no Brasil, é a Telenfermagem, a qual possibilita a capacitação profissional no próprio local de trabalho através do uso de TIC, contribuindo para a EPS. Além disso, por meio da teleconsultoria on-line (síncrona) e off-line (assíncrona), o profissional de enfermagem pode obter uma segunda opinião para auxiliar na tomada de decisão de casos clínicos e de gestão em saúde ${ }^{(12)}$.

$\mathrm{O}$ atendimento remoto pela Enfermagem inclui as chamadas telefônicas, o monitoramento remoto, as fotografias e os vídeos. As chamadas telefônicas foram o primeiro método à distância utilizado para o atendimento de Enfermagem e continua sendo um componente essencial do cuidado em vários ambientes. O monitoramento remoto envolve o uso de dispositivos eletrônicos chamados periféricos, buscando-se capturar dados de saúde do paciente e sintomas para uma intervenção precoce. Constituem-se dispositivos periféricos: os manguitos de pressão arterial, os monitores de glicose e os dispositivos de oximetria de pulso(11).

Assim, considera-se o Telessaúde uma importante ferramenta para o enfermeiro, facilitando-se a sua atuação, principalmente no atual período de pandemia da COVID-19. Contudo, a comunicação à distância pode exercer influência no tempo necessário para uma interação de qualidade, na satisfação dos envolvidos e nas limitações relacionadas à comunicação não verbal ${ }^{(13)}$. Um estudo ${ }^{(13)}$ com o objetivo de avaliar a percepção do enfermeiro referente à comunicação interpessoal no cuidado por Telessaúde demonstrou que os mesmos percebem este tipo de comunicação como um desafio, principalmente devido à dificuldade de percepção de sinais não verbais e que há necessidade de abordagem das novas tecnologias de comunicação e interação na formação profissional.

A pandemia da COVID-19 tem evidenciado as contribuições do Telessaúde para a assistência de Enfermagem. Contudo, deve-se avaliar constantemente os impactos da incorporação de novas tecnologias no cuidado de Enfermagem ${ }^{(13)}$ e considerar uma preparação educacional ampliada e consciente das melhores práticas em Telessaúde, com base em evidências científicas ${ }^{(10)}$, para a garantia de uma assistência de qualidade.

\section{REFERÊNCIAS}

1- Ge H, Wang X, Yuan X, Xiao G, Wang C, Deng T, et al. The epidemiology and clinical information about COVID-19. Eur J Clin Microbiol Infect Dis. 2020;39(6):1011-9. DOI: 10.1007\%2Fs10096-020-03874-z 
2- Harapan H, Itoh N, Yufika A, Winardi W, Keam S, Te H, et al. Coronavirus disease 2019 (COVID-19): A literature review. J Infect Public Health 2020;13(5):667-73. DOI: 10.1016\%2Fj.jiph.2020.03.019

3- Oliveira WK, Duarte E, França GVA, Garcia LP. Como o Brasil pode deter a COVID-19. Epidemiol Serv Saúde 2020;29(2):e2020044. DOI: 10.5123/s1679-49742020000200023

4- Vidal-Alaball J, Acosta-Roja R, Hernández NP, Luque US, Morrison D, Narejos Pérez S, et al. Telemedicine in the face of the COVID-19 pandemic. Aten Primaria 2020;52(6):418-22. DOI: 10.1016/j.aprim.2020.04.003 5- Zanotto BS, Etges APBS, Siqueira AC, Silva RS, Bastos C, Araújo AL, et al. Avaliação econômica de um serviço de telemedicina para ampliação da atenção primária à saúde no Rio Grande do Sul: O microcusteio do Projeto TeleOftalmo. Ciênc Saúde Coletiva 2020;25(4):1349-60. DOI: 10.1590/1413-81232020254.28992019 6- Brasil. Ministério da Saúde. Portaria no 2.546, de 27 de outubro de 2011. Redefine e amplia o Programa Telessaúde Brasil, que passa a ser denominado Programa Nacional Telessaúde Brasil Redes (Telessaúde Brasil Redes). Diário Oficial da União 2011.

7- Damasceno RF, Caldeira AP. Fatores associados à não utilização da teleconsultoria por médicos da Estratégia Saúde da Família. Ciênc Saúde Coletiva 2019;24(8):3089-98. DOI: 10.1590/1413$\underline{81232018248.28752017}$

8- Caetano R, Silva AB, Guedes ACCM, Paiva CCN, Ribeiro GR, Santos DL, et al. Desafios e oportunidades para telessaúde em tempos da pandemia pela COVID-19: Uma reflexão sobre os espaços e iniciativas no contexto brasileiro. Cad Saúde Pública 2020;36(5):e00088920. DOI: 10.1590/0102-311x00088920

9- Pollock K, Setzen M, Svider PF. Embracing telemedicine into your otolaryngology practice amid the COVID19 crisis: An invited commentary. Am J Otolaryngol. 2020;41(3):102490. DOI: https://dx.doi.org/10.1016\%2Fj.amjoto.2020.102490

10- Byrne MD. Telehealth and the COVID-19 Pandemic. J Perianesth Nurs. 2020; 35(5):548-551. DOI: 10.1016\%2Fj.jopan.2020.06.023

11- Mahoney MF. Telehealth, telemedicine, and related technologic platforms. J Wound Ostomy Continence Nurs. 2020;47(5):439-44. DOI: 10.1097/WON.0000000000000694

12- Guimarães EMP, Godoy SCB. Telenfermagem: Recurso para assistência e educação em enfermagem. Rev Min Enferm. 2012 [citado em 15 mar 2020]; 16(2):157-8. Acesso em: http://www.reme.org.br/artigo/detalhes/513

13- Barbosa IA, Silva MJP. Cuidado de enfermagem por telessaúde: Qual a influência da distância na comunicação? Rev Bras Enferm. 2017;70(5):928-34. DOI: 10.1590/0034-7167-2016-0142 